\title{
PERSONALIZED LEARNING-BASED SEGMENTATION OF THORACIC AORTA AND MAIN BRANCHES FOR DIAGNOSIS AND TREATMENT PLANNING
}

\author{
Dime Vitanovski ${ }^{\star \dagger}$, Kristof Ralovich ${ }^{\star}$, Razvan Ionasec ${ }^{\star}$, Yefeng Zheng ${ }^{\star}$, Michael Suehling ${ }^{\star}$, \\ Waldemar Krawtschuk ${ }^{\star}$, Joachim Hornegger ${ }^{\dagger}$ and Dorin Comaniciu ${ }^{\star}$ \\ * Image Analytics and Medical Informatics, Siemens Corporate Research, Princeton, USA \\ ${ }^{\dagger}$ Pattern Recongnition Lab, University of Erlangen-Nuremeberg, Germany
}

Coarctation of the aorta $(\mathrm{CoA})$, is an obstruction of the aortic arch present in $5-8 \%$ of congenital heart diseases. For children older than a year, CoA is increasingly treated by aortic stenting instead of surgical repair. In pediatric cardiology, CMR is accepted as the standard non-invasive imaging modality to assess the aortic arch in it's entire spatial context [1]. Interpreting such 3D datasets are required to assess the underlying anatomy during both diagnosis and therapy planning phases. However this process is time consuming and varies with operator skills. Within this study we propose - for the first time in our knowledge - a method of automatic segmentation of the lumen of thoracic aorta and main branches. The personalized model of the aorta and the supra-aortic arteries, automatically estimated from 3D CMR data, will provide better understanding of the complexity of pathology and assist the cardiologist to choose the best treatment and timing of repair. A hierarchical framework based on robust machinelearning algorithms is proposed to estimate the personalized model parameters. Experiments throughout 212 3D CMR volumes demonstrate model estimation error of $3.24 \mathrm{~mm}$ and average computation time of $8 \mathrm{sec}$. combined with clinical evaluation on 32 patients.

\section{INTRODUCTION}

Coarctation of the aorta and bicuspid aortic valve are common congenital heart defects which affect $5-8 \%$ of the annual live births in USA [2]. Surgery is considered as "gold standard" for CoA and Bicuspid Aortic Valve (BAV) treatment, and recently balloon angioplasty and stenting are investigated for effectiveness [3] and increasingly applied after the neonatal period.

Patients with successful coarctation repair during their childhood are still confronted with increased probability of cardiovascular complications: restenosis, residual and recurrent systemic hypertension, aortic aneurisms and dissections, hemopericardium and associated aortic valve malformations, and premature coronary artery disease $[4,5]$. These clinical findings underline the important need for accurate anatomical evaluation of the aorta during diagnosis, treatment and follow-up procedures based on Cardiac Magnetic Resonance (CMR) imaging [6]. To this end, we propose an automatic and accurate method for segmenting the thoracic arterial tree (including the ascending aorta, transverse arch, distal descending aorta and three supra aortic arteries), to allow fast and reproducible assessment of the lumen. The inclusion of the supra aortic arteries in our segmentation was dictated by their role played in CoA situations: these branches of the aorta supply the head and arms with oxigenated blood and account for $30-40 \%$ of the blood flow leaving the aorta, and thus are subject to higher blood pressures before repair. The segmented vessel models allow further automated lumen measurements (for e.g. catheter selection) severity assessment of coarctation, dilation and aneurisms. Further possibilities include generation of boundary conditions for patient-specific hemodynamics simulations of the region. To the best of our knowledge, this is the first time in literature to present automatic segmentation of aorta and supra aortic arteries from CMR.

Literature contains existing work from multiple authors addressing delineation of the aorta.

Zheng et al. [7] proposed an automatic detection approach for transcatether aortic valve implantation procedures, focusing on pre- and intra-operative contrast enhanced CT and DynaCT images. Despite remarkable robustness, this work is not considering the supra aortic arteries. Recently an extensive study was carried out, exclusively focusing on the carotid artery [8] bifurcation, concluding that accurate automatic segmentation is feasible. Their results are limited to high resolution CTA images. There are a few approaches that employ CMR images[9, 10]. Both methods are semi-automatic, required user interaction and do not include the supra-aortic arteries in their final model.

Within this paper we present a personalized model of the aorta and the supra-aortic arteries automatically estimated from CMR data acquired with different protocols and sequences. A two stage hierarchical machine-learning based approach is proposed to first detect the model of the aorta and then the supra-aortic arteries (Sec. 2). For each hierarchical layer robust detectors are trained with PBT[11] and 3D 


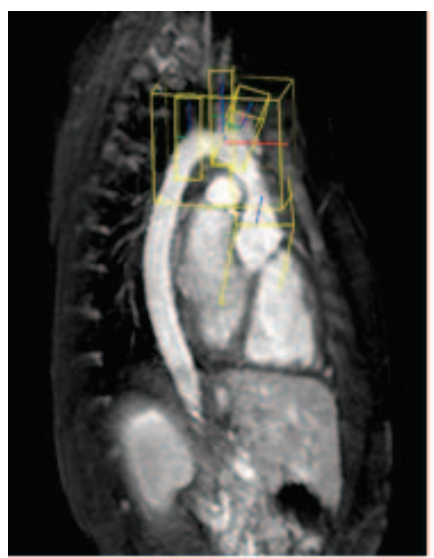

a)

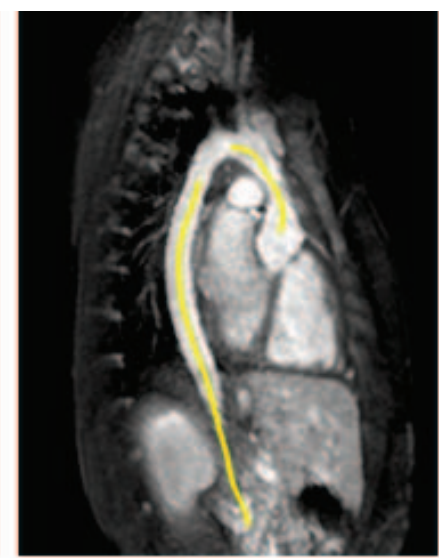

b)

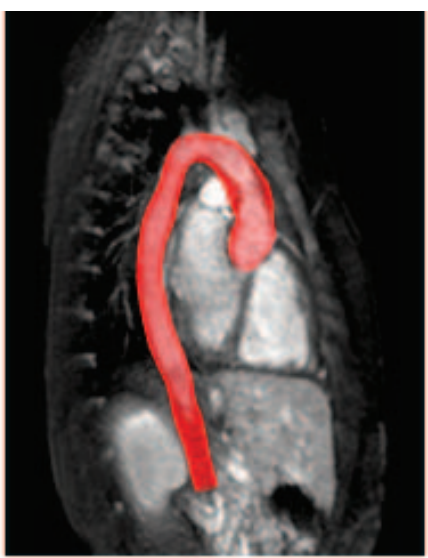

c)

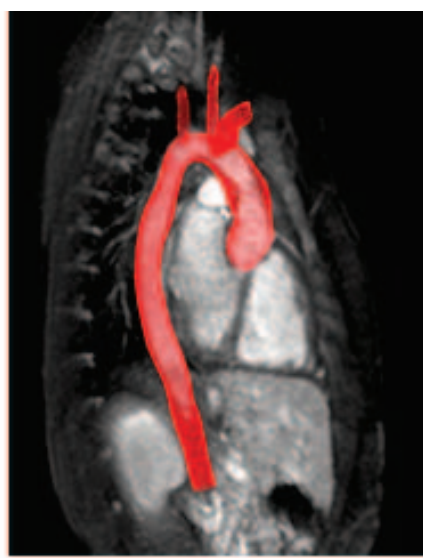

d)

Fig. 1. Lumen segmentation pipeline: a) anatomy localization (bounding box) b) centreline extraction c) non-rigid boundary refinemet d) complete aortic model composed by the aorta and the supra-aortic arteries

Haar and steerable features [12]. The coarse-to-fine model estimation process starts by first localizing the aortic root and arch, followed by 2D circle tracking (Fig. 2.2) and non-rigid boundary refinement (Fig. 1). In the second phase the search ranges of the supra-aortic detectors are constrained by the previously estimated models. Finally, we employ a sequence of forward and backward projection to/from Eulerian representation of the separately detected models to retrieve the composite Lagrangian arterial tree geometry (Fig. 1(d)).

The accuracy of our method is demonstrated on a set of 212 3D volumes (99 patients) with wider range of morphological and pathological variations (Sec. 3). In Sec. 3.1 we demonstrate the significant correlation between measurements manually performed by our clinical collaborators and derived from our model.

\section{METHOD}

\subsection{Global Anatomy Localization}

For each anatomy (aortic root, aortic arch, brachio artery, left common carotid, left subclavian artery) we define a corresponding bounding box $\left(\theta_{\text {root }}, \theta_{\text {arch }}, \theta_{\text {brachio }}, \theta_{\text {left_common }}\right.$, $\left.\theta_{\text {left_subclavian }}\right)$ parameterized by an affine transformation $\theta=\left(X, Y, Z, \alpha, \beta, \chi, S_{x}, S_{y}, S_{z}\right)$. The position of each bounding box is computed as the centre of gravity of the corresponding manually annotated mesh. Correspondingly, the orientation is given by the first three PCA eigenvectors which correspond to the largest eigenvalues and the scale is computed by searching for the furthest mesh point along the orientation axis.

We formulated the anatomy localization task as a classification problem and trained discriminative detectors relying on robust features. Efficient learning in high dimensional space is performed by marginalizing the search space by means of
MSL into subspaces which results in gradual increase of degrees of detector freedom:

$$
\Sigma_{1} \subset \Sigma_{2} \subset \cdots \subset \Sigma_{n}=\Sigma
$$

Consequently, the parameter estimation problem is splitted into three steps where classifiers, parameterized by the current subset of the affine values, are sequentially trained on the subspaces: position, position-rotation and full affine transformation. By decomposing the original search space as follows:

$$
\begin{aligned}
& \Sigma_{1}=\left(t_{x}, t_{y}, t_{z}\right) \\
& \Sigma_{2}=\left(t_{x}, t_{y}, t_{z}, \vec{\alpha}_{x}, \vec{\alpha}_{y}, \vec{\alpha}_{z}\right) \\
& \Sigma_{3}=\left(t_{x}, t_{y}, t_{z}, \vec{\alpha}_{x}, \vec{\alpha}_{y}, \vec{\alpha}_{z}, s_{x}, s_{y}, s_{z}\right)
\end{aligned}
$$

the target posterior probability can be expressed as:

$$
\begin{aligned}
& p(\theta(t) \mid I(t))=p\left(t_{x}, t_{y}, t_{z} \mid I(t)\right) \\
& p\left(\vec{\alpha}_{x}, \vec{\alpha}_{y}, \vec{\alpha}_{z} \mid t_{x}, t_{y}, t_{z}, I(t)\right) \\
& p\left(s_{x}, s_{y}, s_{z} \mid \vec{\alpha}_{x}, \vec{\alpha}_{y}, \vec{\alpha}_{z}, t_{x}, t_{y}, t_{z}, I(t)\right)
\end{aligned}
$$

A further speed improvement is achieved by using a pyramidalbased coarse-to-fine approach and searching in a subsampled low-resolution $(3 \mathrm{~mm})$ image first.

\subsection{Personalized Model Detection}

Aortic Model Estimation. Due to the high anatomic variation of the aorta, especially the ascending/descending part caused by pathologies and differences in age we propose a part-based approach for detecting the aortic model by splitting the aorta in four patches: aortic root, ascending aorta, aortic arch and descending aorta. First, the detected bounding boxes $\theta_{\text {root }}, \theta_{\text {arch }}$ initialize the surface by projecting a mean shape $M_{\text {root }}, M_{\text {arch }}$ averaged over the training data set, into the corresponding bounding box. The transformed mean 
model provides accurate global fitting into the patient's specific anatomy. However, further local processing for precise object delineation is required. A boundary detector trained by PBT is applied along the normal of each discrete 3D boundary point to test a set of hypothesis and move the surface towards the position with the highest probability response. Smooth boundary is obtained by projecting the detected model into the pre-computed PCA shape space [13]. The same approach is applied to estimate the aortic arch.

A tracking algorithm is used to deal with the different variation of the length of the ascending and the descending patches. As the intersection of both patches with an image slice is close to circle, we trained a 2D circle detector to estimate primitive circular structures between the aortic root and the arch (ascending patch). The descending part is estimated by tracking circles starting from the aortic arch towards the patient's feet. The algorithm stops if the volume border has been reached. At each image slice the trained 2D detector assigns each voxel with a probability of being on the circumference of the lumen. In order to reduce outliers, the search ranges of the detector are constrained by the size of the previously detected circle.

Fusing all separately estimated aortic patches together into a single model, we get an initial, close to the true boundary, estimate of the whole aorta. As circle do not precisely capture local deformation of the aorta, further non-rigid refinement by trained detector is performed for precise object delineation.

The robustness of the tracking method is shown by successfull handling of post-stenting images that inhibit CMR signal dropout at the location of the stent (the dark region illustred in Fig. 2.2).
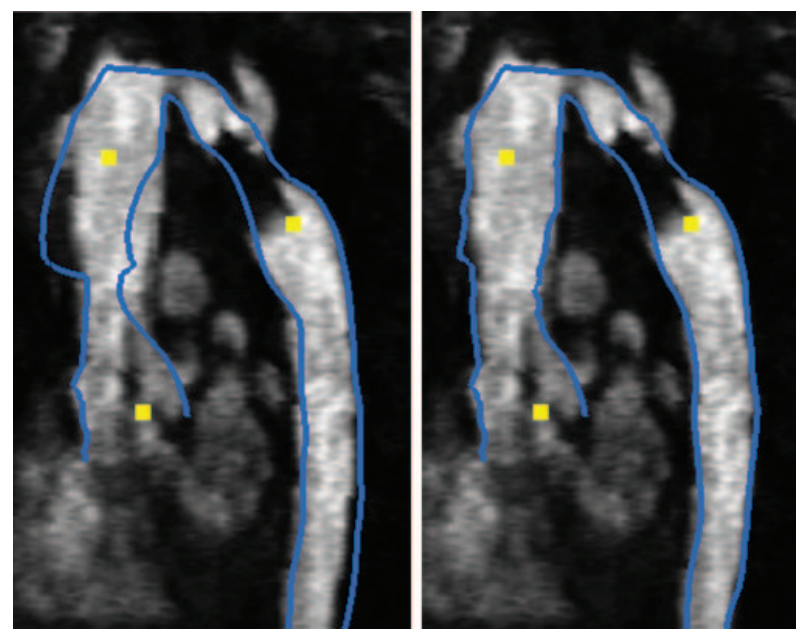

Fig. 2. Left: ascending and descending aortic patches after 2D circle detection. Right: final non-rigid refined aortic model

Supra-Aortic Model Estimation. The supra-aortic ar- teries are estimated in same manner by first localizing each artery separately with a bounding box, placing a mean model within the estimated box and applying a trained boundary detector for precise object delineation. However, due to the high variation of the arteries regarding pose and shape, detecting them with constraints is beneficial. Therefore, previously estimated anatomies are used to impose constraints to the detector: we use the position and orientation of the aortic arch to crop the volume and reduce the search ranges of the brachiocephalic artery detector. Testing a set of hypothesis in such sub-volume reduces significantly the number of outliers and false positives. The search space for each other supra-aortic artery is gradually reduced by the aortic arch and the previously detected artery.

\section{EXPERIMENTAL RESULTS}

We demonstrate the performance of the proposed hierarchical detection framework on 99 Patients (212 3D CMR Volumes). Data were collected from 5 different hospitals and acquired using heterogeneous CMR acquisition protocols/sequences with different CMR devices (Siemens, Philips and GE).

Each volume in the data set is associated with annotation, manually generated, which is considered as ground truth for the learning algorithm. The personalized complete aortic model estimation accuracy was evaluated by using the pointto-mesh metric. For each point of the estimated model we compute the Euclidean distance to the closest point of the associated, manually generated, ground-truth model. Table 1 summarizes the estimation performance for each detection step.

\begin{tabular}{|c|c|c|c|c|}
\hline mm & Mean & Median & $80 \%$ & $90 \%$ \\
\hline aorta & $2.29 \pm 1.74$ & 1.95 & 2.37 & 2.67 \\
\hline brachiocephalic & $3.40 \pm 1.89$ & 2.90 & 4.33 & 5.60 \\
\hline left common & $4.59 \pm 3.58$ & 3.16 & 5.99 & 8.44 \\
\hline left subclavian & $4.64 \pm 3.33$ & 3.06 & 6.19 & 8.90 \\
\hline supra-aortic & $4.21 \pm 2.90$ & 3.04 & 5.05 & 7.64 \\
\hline complete model & $3.24 \pm 2.32$ & 2.49 & 3.72 & 5.15 \\
\hline
\end{tabular}

Table 1. Detection performance accuracy (in $\mathrm{mm}$ )

\subsection{Clinical Evaluation}

The quantitative capabilities of our system are demonstrated on 32 patients with aortic anomalies (age: 5-36 years, 17 with CoA and 15 with BAV and ascending aorta dilation) by comparing a set of morphological measurements[6] automatically derived by our personalized model to measurements manually extracted from our clinical collaborators. The aortic min and max diameter were measured from 3D SSFP CMR sequence at five segments: aortic sinus (AS), sino-tubular junction (STJ), ascending aorta (AAO), transverse arch (TA), and 
descending aorta (DA). Statistical results significantly correlated ( $p<0.001, r=0.94$ ) between model-based and manual measured min and max diameters. Table 2 summarizes the mean measurement errors for each segment separately.

\begin{tabular}{|c|c|c|c|}
\hline $\mathrm{mm}$ & AS & STJ & AAO \\
\hline $\min$ & $1.61 \pm 0.9$ & $2.07 \pm 1.5$ & $1.61 \pm 1.9$ \\
\hline $\max$ & $1.56 \pm 1.3$ & $1.28 \pm 1.0$ & $1.56 \pm 1.3$ \\
\hline \multirow{4}{*}{$\operatorname{mm}$} & \multicolumn{2}{|c|}{ TA } & DA \\
\cline { 2 - 4 } & $\min$ & $1.70 \pm 1.2$ & $0.8 \pm 0.5$ \\
\cline { 2 - 4 } & $\max$ & $1.34 \pm 1.1$ & $0.92 \pm 0.6$ \\
\hline
\end{tabular}

Table 2. Comparison between manual and model-based derived measurements

These measurements are crucial in clinical settings for optimal treatment selection and decision making. The modebased derived size of each segment and the exact location of the coarctation will facilitate the decision on the right timing and type of surgical approach in CoA and (AAO) dilation due to BAV. Especially in patients with BAV and dilatation of the proximal AAO, where several surgical options are influenced by the location and extent of AAO dilation and the functional status of the valve, the model-based assessment of the aortic anatomy and morphology will advance the choice between Bentall versus Yacoub or David surgical approach [14].

\section{CONCLUSION}

This paper proposes a method for automatic estimation of personalized, complete aortic model which includes the aorta and the supra-aortic arteries. Two stage hierarchical approach was introduced for the coarse-to-fine model parameter estimation. In each stage efficient and robust machine-learning algorithm were presented to guide the segmentation. The estimated complete aortic model is utilized for better morphological and physiological assessment of the aortic anatomy. Accurate and precise model-based derived measurements are facilitating for optimal treatment selection, decision making and timing of repair. In future work, we would like to extend the method to 4D, temporal data and investigate explicit stent localization.

\section{REFERENCES}

[1] S.B. Greenberg and et al., "Coarctation of the aorta: diagnostic imaging after corrective surgery," Journal of Thoracic Imaging, vol. 10, no. 1, pp. 36-42, 1995.

[2] W. Rosamond and et al., "Heart disease and stroke statistics-2007 update: a report from the american heart association statistics committee and stroke statistics subcommittee," Circulation, vol. 115, no. 5, pp. 69-171, 2007.
[3] R.E. Ringel and K. Jenkins, "Coarctation of the aorta stent trial (coast)," 2007.

[4] H.O. Toro-Salazar and et al., "Long-term follow-up of patients after coarctation of the aorta repair," The American Journal of Cardiology, vol. 89, no. 5, pp. 541-547, 2002.

[5] O. Jose Maria and et al., "Risk factors for aortic complications in adults with coarctation of the aorta," American College of Cardiology, vol. 44, no. 8, pp. 1641-7, 2004.

[6] A.D. Pasqua and et al., "Comparison of contrast and noncontrast magnetic resonance angiography for quantitative analysis of thoracic arteries in young patients with congenital heart defects," Ann Pediatr Cardiology, vol. 4, no. 1, pp. 36-40, 2011.

[7] Y. Zheng and et al., "Automatic aorta segmentation and valve landmark detection in c-arm ct: Application to aortic valve implantation," in Medical Image Computing and Computer Assisted Intervention, 2010.

[8] K. Hameeteman and et al., "Evaluation framework for carotid bifurcation lumen segmentation and stenosis grading.," Medical Image Analysis, vol. 15, pp. 477488, 2011.

[9] F. Zhao and et al., "Automated 4d segmentation of aortic magnetic resonance images," in The 17th British Machine Vision Association, Sept. 2006, vol. 1, pp. 247257.

[10] E. Flehmann and et al., "Towards patient specific catheter selection: computation of aortic geometry based on fused mri data," in Proceedings of the 6th international conference on Functional imaging and modeling of the heart, 2011, pp. 145-152.

[11] Z. Tu, "Probabilistic boosting-tree: Learning discriminativemethods for classification, recognition, and clustering," in ICCV 2005, 2005, pp. 1589-1596.

[12] Z. Yefeng and et al., "Four-chamber heart modeling and automatic segmentation for 3-d cardiac ct volumes using marginal space learning and steerable features," IEEE Transactions on Medical Imaging, vol. 27, no. 11, pp. 1668-1681, Nov. 2008.

[13] T. Cootes, "Active shape models-their training and application," Computer Vision and Image Understanding, vol. 61, no. 1, pp. 38-59, 1995.

[14] H. Bentall and A. De Bono, "A technique for complete replacement of the ascending aorta," Thorax, vol. 23, no. 4, pp. 3389, 1968. 